\title{
Sobre casas, ventanas y miradas: Una cita con José Donoso y Henry James
}

About houses, windows and views: An appointment with José Donoso and Henry James

\section{Sebastián Schoennenbeck Grohnert}

Pontificia Universidad Católica de Chile. Santiago, Chile. sschoenn@uc.cl

\section{RESUMEN}

En la obra de José Donoso, la representación de la casa es violentada por discursos externos que irrumpen en el espacio doméstico y cerrado, alterando al sujeto que lo habita. Sin embargo, en El obsceno pájaro de la noche y en algunos ensayos del autor, la casa supone también un lenguaje metaliterario que cita la reflexión de Henry James en torno a lo que él denominó como la casa de la ficción. En efecto, la casa es leída como metáfora de composición, ya que supone la presencia de un sujeto que representa la realidad observada a través de la ventana.

Palabras claves: Casa, sujeto, mirada, representación, ficción.

\section{ABSTRACT}

In José Donoso's work, the representation of the house is done violence to by external discourses that break into the closed domestic space, altering the subject that inhabits it. However, in El obsceno pájaro de la noche and in some other author's essays, the house supposes as well a methaliterary language that quotes Henry James' reflection

* Este artículo forma parte de la investigación posdoctoral financiada por el Fondo Nacional de Desarrollo Científico y Tecnológico de Chile titulada "La ruta anglosajona en la poética de José Donoso", Proyecto Fondecyt No 3095004. 
of what he defines as the house of fiction. In effect, the house is read as a metaphoric composition, given its assumption of the presence of the subject that represents the reality observed through a window.

Keywords: House, subject, view, representation, fiction.

Recibido: 06-09-2010. Aceptado: 16-11-2010.

T a casa es uno de los espacios más recurrentes en el imaginario de José Do_noso (1924-1996). Desde su primera novela, Coronación (1956), hasta la última obra póstuma titulada Lagartija sin cola (2007), la casa se presenta una y otra vez bajo diferentes versiones. Palacios, prostíbulos, pensiones, casas de playa y de poetas constituyen una serie de apariencias a través de las cuales se representa el microcosmos de lo cotidiano, una imagen poética que, en la obra donosiana, traiciona su promesa concerniente al recuerdo de un pasado que asegure la integridad del sujeto ${ }^{1}$. Con respecto a una de las novelas póstumas de J. Donoso, Julio Ortega (2010) comenta: "La visión de una Casa que convoque todos los tiempos vividos en un convivio liberado de la sociedad y sus demandas, se demuestra como melancolía" (476). En efecto, la casa donosiana no fortalece los límites que separan lo público de lo privado. Tampoco provee de una protección tal del espacio interior que impida la irrupción de las fuerzas extrañas provenientes del mundo exterior. J. Donoso nos presenta entonces una casa que tarde o temprano es alterada, es decir, lo otro transforma y sustituye al habitante original y con ello su ethos. Pienso, por ejemplo, en la lectura de Ana María Dopico (2001), para quien la obra de J. Donoso señala proféticamente los quiebres sociales de un contexto de represión dictatorial que atañen incluso a la mayoría de los hogares burgueses. Para la estudiosa, en El obsceno pájaro de la noche, la casa es el espacio donde se efectúa un delito pesadillesco por efecto de una ley violenta: "The novel offers a vision of monstrosity, breakdown and entombment, marking the domestic entrampment..." (325). El devenir de la casa yuxtapone entonces un orden previo y uno nuevo, orden en el cual el sujeto trastornado no se reconoce, puesto que su habitar ya no tiene nada de habitual. El hábito de vivir se ha roto.

\footnotetext{
${ }^{1}$ Así, al menos, podría leerse los dos primeros capítulos titulados "La casa. Del sótano a la guardilla" y "La casa y el universo" de La poética del espacio de Gastón Bachelard (1975). Desde la perspectiva de la fenomenología de la imagen, el autor propone la casa como un cosmos y un refugio del sujeto y de su pasado.
} 
Lo obsceno es el rasgo más definitorio de la casa donosiana. Dicha obscenidad degrada todo aquello que el espacio doméstico puede ser o llegar a ser. En efecto, la casa puede relatar una historia familiar al resguardar una serie de objetos que estimulan el recuerdo del pasado heredado. Espacio de socialización, la casa, al estar habitada por el grupo familiar, deviene también hogar, lugar donde se forma y produce discursivamente, por ejemplo, al sujeto nacional ${ }^{2}$. A su vez, la casa es el reflejo unificador de los fragmentos del sujeto que la habita, ya que los objetos que la decoran o que construyen la cotidiana funcionalidad doméstica acusan los gustos, intereses, costumbres y orígenes generacionales, sociales y genéricos de toda una personalidad. Pero por sobre todo, la casa es el lugar de la intimidad, porque, en tanto espacio privado, se opone al ámbito de la vida social, de la ciudad y del trabajo, o sea, a los espacios donde se deviene sujeto público. De este modo, la casa, gracias a sus diferentes compartimentos, presenta un camino gradual desde lo público a lo privado. El hall de entrada, el salón y el comedor serían los espacios de transición entre dos ámbitos opuestos: por un lado, el carácter público de la calle, la plaza, la feria, el bar y el trabajo y, por el otro lado, la intimidad máxima que el sujeto obtiene en el dormitorio, lugar de distensión en el que el cuerpo, ya liberado de la regla social, se sacude de las máscaras y de otros artificios que la cultura le otorga e impone.

Mientras la casa como espacio familiar remite al proceso de socialización más inmediato, el tipo de casa que revela, por el contrario, el mayor grado de intimidad es aquélla habitada por un sujeto asiduo a la soledad. Es el caso, por ejemplo, de la casa de Andrés Ávalos, uno de los personajes principales de Coronación (1957). Aquí el transcurrir de los días se desliza en un sutil encierro protector, en un aislamiento con respecto al mundo moderno, en un apego al pasado que intenta conservar un viejo mundo y en un desapego al presente y al futuro. En la casa del soltero, el sujeto se des-socializa, perdiendo su vínculo con el mundo de afuera. Su ciudadanía se vuelve un exilio en el que se contempla el silencio de los objetos rechazados por el orden actual. La intimidad de esta casa no sólo radica en la soledad de quien la habita, sino también en el diálogo secreto del coleccionista y sus cosas, ya que el primero también se vuelve un objeto de la mirada de sus bibelots y retratos. Es esta relación recíproca y desconcertante la que Donoso revela con toda su extravagante intimidad, transformando ese espacio original en una representación obscena. En este sentido, J. Donoso nos recuerda que

${ }^{2}$ Sobre la relación alegórica familia-nación en la narrativa hispanoamericana del siglo XIX, ver Sommer, Doris. 2004. Ficciones fundacionales. Traducción de José Leandro Urbina. Colombia: Fondo de Cultura Económica. 
toda representación es un acto obsceno: ante los espectadores se presenta, se muestra y se señala con un dedo tenebroso lo que estaba fuera de los alcances de miradas ajenas. Aquella intimidad que por ser tal estaba fuera de escena es ahora develada descarnadamente.

Una manera de problematizar la casa en la obra de J. Donoso es pensar en aquellos antecedentes que se refieren a tal espacio y que podemos encontrar al indagar en las lecturas del autor. Me interesa, por cierto, una biografía de Donoso no sólo como sujeto social, sino también como lector en la medida que un registro de las apropiaciones de las tradiciones culturales con las cuales dialogó devela, más que una poética autoral, una poética del género literario que cultivó y que podría ser definida, en este caso, como una poética de la novela ${ }^{3}$. Pensemos también que el registro de las lecturas habla no sólo del objeto leído, sino también del sujeto que registra. Tal como lo ha afirmado S. C. Fazio (2010), la escritura crítica del lector es una autobiografía, un modo delegado de autoconfiguración en el que se da a conocer no sólo una selección biográfica y juicios estéticos, sino también los intereses y el temperamento del sujeto en cuestión.

Dado lo anterior, la figura de Henry James (1843-1816) asoma como un fantasma en nuestra empresa de visualizar a J. Donoso como un lector que hereda ciertos imaginarios domésticos para luego ampliarlos a lo largo de su obra. Es sabida la gran importancia que tuvo Henry James en la formación del escritor chileno tal como sus ensayos, entrevistas y cuadernos de notas lo atestiguan ${ }^{4}$. En ambos autores, la casa se manifiesta en dos planos: en el de la reflexión metaliteraria y en el de la representación propiamente tal de dicho espacio como elemento que altera al sujeto. Este último plano puede ser identificado con un proceso de destrucción del espacio llevado a cabo por una palabra que, al igual que la palabra mítica de El obsceno pájaro de la

\footnotetext{
${ }^{3}$ Estoy pensando en la definición de poética dada por Todorov, quien, rescatando una dimensión histórica, apela al estudio de la evolución de los géneros literarios. Ver Todorov, T. 1971. "Poética estructural". En Qué es el estructuralismo. Traducción de Ricardo Pochtar. Buenos Aires: Losada. 103-171.

${ }^{4}$ Con respecto a los ensayos y entrevistas, se puede consultar los siguientes títulos: Donoso, José. 1998. "El maestro cumple setenta". Artículos de incierta necesidad. Selección de Cecilia García Huidobro Santiago: Alfaguara, pp. 402-409.

McMurray, George. 1975. "Interview with José Donoso”, en Hispania 58, pp. 391-393.

Carminatti, Graciela. 1980-1. "Entrevista con José Donoso", en Revista de la Universidad de México 35, pp. 56-58.

En cuanto a sus cuadernos de notas, me refiero a los manuscritos que se guardan en la Universidad de Iowa y Princeton. Los sitios web de ambas colecciones son los siguientes: http://diglib.princeton.edu/ead/eadGetDoc.xq?id=/ead/mss/C0099.EAD.xml http://www.lib.uiowa.edu/spec-coll/MSC/ToMsc350/MsC340_Donoso/Donoso_340.htm
} 
noche, es "el germen del terror" (Valdés, 1975: 135). En el otro plano, por el contrario, se presenta un proceso de creación sobre el cual se medita, dando lugar al discurso metaliterario.

\section{LA CASA Y LA REFLEXIÓN METALITERARIA}

En la reflexión de ambos autores, la casa desempeña un rol metafórico a través de la cual se describe el arte de la ficción. En el caso de Henry James, la casa equivale a la conciencia, espacio en el cual se dramatiza la acción que se relata. Al respecto, Edgardo Cozarinsky (1964) sintetiza la obra del autor anglosajón como la "... dramatización de un hecho mediante su reflejo en la conciencia de un personaje, la búsqueda de puntos de vista cuyos privilegios y limitaciones darán forma a la narración" (11). En este sentido, la acción narrada en gran parte de la obra de James no es aquella que acontece objetivamente, sino más bien el reflejo de esta misma en una conciencia. O sea, la realidad que acontece y aparece en el mundo jamesiano está mediada por un punto de vista. De este modo, el narrador, ya sea personaje que participa en la historia o voz impersonal, comparte la conciencia y el conocimiento del personaje o de los personajes elegidos para tal focalización. Al respecto, José Donoso (1998a) recuerda las palabras de su maestro en un ensayo titulado "El discreto encanto de los prólogos":

Lo que un novelista dice debe siempre estar presente en el conocimiento específico de uno de los personajes sobre el hecho; su insistencia en el detalle, en lo específico, en lo no nebuloso; lo que en el arte se 'dice' no es 'presentado', lo que no se 'presenta' no es vivido, lo que no es vivido no es una 'representación', y lo que no es una 'representación' no es arte (397).

La idea de concebir metafóricamente la casa como conciencia encuentra su más convincente exposición en el prólogo que el mismo James (1973a) escribió a su novela Retrato de una dama (1881). Para el escritor norteamericano, la casa, gracias a sus múltiples ventanas, es el lugar desde donde se mira "el escenario humano" o la realidad. De este modo, el autor nos permite identificar al sujeto que observa y a las ventanas de la casa a través de las cuales dicha observación tiene lugar con el lenguaje o la forma mientras que la realidad observada o representada es el tema o asunto. Por ende, la ventana figura como un umbral que mediatiza los sentidos del que mira, determinando la mirada a partir de su forma. Sujeto y ventana conforman 
así una unidad que determina la representación narrativa:

La casa de la ficción, en suma, no tiene una sino un millón de ventanas... más bien, un número incontable de posibles ventanas; cada una de las cuales ha sido abierta, o puede aún abrirse, en su extenso frente, por exigencia de la visión individual y por presión de la voluntad individual. Esas aberturas, de forma y tamaño desigual, dan todas sobre el escenario humano, de modo tal que podríamos esperar de ellas una mayor semejanza de noticias de la que hallamos. Pero cuando más, son ventanas, meros agujeros en un muro inerte, desconectadas, encaramadas en lo alto; no son puertas articuladas abiertas directamente sobre la vida. Tienen una característica propia: detrás de cada una de ellas se yergue una figura provista de un par de ojos, o al menos de prismáticos, que constituye, una y otra vez, para la observación, un instrumento único que asegure a quien lo emplea una impresión distinta de todas las demás. El y sus vecinos están contemplando la misma representación, pero uno ve más donde otro ve menos, uno ve negro donde el otro ve blanco, uno ve grande donde el otro ve pequeño, uno ve tosco donde el otro ve refinado. Y así sucesivamente; por fortuna no puede decirse sobre qué no se abrirá una ventana, para un par de ojos particular; "por fortuna" en razón, precisamente, de esa incalculabilidad de alcance. El ancho campo, el escenario humano, es la "elección del asunto"; la abertura, sea amplia o abalconada o baja o como un tajo, es la "forma literaria"; pero, juntas o separadas, son nada sin la presencia del observador; dicho con otras palabras, sin la conciencia del artista: decidme cómo es el artista y os diré de qué ha tenido conciencia (62).

Dado lo anterior, la forma de la novela está dada por la mirada que determina las apariencias de los objetos observados. A su vez, la manera de mirar depende de cómo la ventana sea. La atención por parte del autor se ha desviado entonces desde el asunto observado a la observación misma puesta ya en jaque al estar interferida, obstaculizada o mediada. En efecto, la mirada del personaje es interferida por la naturaleza de la ventana y, en segundo lugar, por la mirada del narrador que mira cómo los personajes miran a través de la ventana. Sin embargo, el "narrador personal" de James representa tales miradas transparentándose como el vidrio de una ventana, ya que, cuando toma la voz en una tercera persona, no contamina con provecho propio la mirada de la conciencia en la cual se focaliza, dejando a un lado opiniones y juicios de valor. Así, su mediación es simulada.

Víctor I. Stoichita (2005) ha trabajo el tema de la representación de la 
mirada en la pintura, llegando incluso a vincular su objeto de estudio con E. Zola y H. James. Gracias a la observación de algunas pinturas impresionistas tales como "El ferrocarril" de Manet, Stoichita hablará del tópico de la "mirada filtrada". Entre el exterior y el sujeto que mira, ha tomado lugar una ventana con un velo o una pantalla sobre la cual se proyecta la realidad observada. Es decir, el autor indica que la mirada ya es representación del objeto al que se enfrenta y que esa representación/mirada deforma o altera el referente original (el objeto observado), impidiendo al sujeto conocer al menos visualmente una realidad inalterada por su propia subjetividad. El velo, la balaustrada o los barrotes de la ventana entorpecen el espacio que separa el ojo del objeto. Así, la mirada que transita por tal espacio es interferida, contaminada o enriquecida, viéndose impedida de encontrar la realidad original que se pretende aprehender. E. Zola, en su ensayo titulado "La pantalla" (1866) lo explica de la siguiente manera:

Cualquier obra de arte es como una ventana abierta a la creación; existe en el encuadre de la ventana una especie de pantalla transparente, a través de la cual se perciben los objetos más o menos deformados, sufriendo cambios más o menos sensibles en sus líneas y en su color. Estos cambios corresponden a la naturaleza de la pantalla. No se tiene la creación exacta y real, sino la creación modificada por el medio a través del cual pasa la imagen. Vemos la creación en una obra a través de un hombre, un temperamento, una personalidad. La imagen que se produce sobre esta pantalla de nueva especie es la representación de las cosas y de las personas situadas más allá, y esta reproducción no puede ser fiel, pues cambia cada vez que una nueva pantalla viene a interponerse entre nuestro ojo y la creación (citado por Stoichita, 2005: 26).

La ventana es entonces una trasparencia velada. Sobre la superficie del cristal, el velo, con su doble y contradictoria función de develar y ocultar, se ha vuelto interrupción de un tránsito ocular. Cadena de interferencias o de mediaciones que se interponen entre el espectador y el espectáculo, la conciencia, el velo y la ventana constituyen esa arquitectura con la cual James crea sus composiciones regidas bajo el principio de la intermediación:

La teoría de la literatura ha demostrado lo importante que son "las figuras intermedias" tanto para el arte narrativo de Zola (en Francia) como para el de Henry James (en el círculo anglosajón). Estas figuras, a veces periféricas, a veces centrales, en función de la importancia que el autor confiera a lo 
que desea describir, posibilitan eludir la descripción directa o la instancia autorial. Mediante el uso de esas figuras el lector participa de la descripción realizándola, por decirlo así, "a través de los ojos" de un personaje de la novela (Stoichita, 2005: 27).

Las referencias de José Donoso a la casa a lo largo de sus reflexiones metaliterarias comparten muchos de los rasgos con los cuales James pensó aquel espacio a la hora de describir la novela. En primer lugar, el autor chileno establece una relación metafórica entre la novela y la casa en el sentido que ambas son composición y, por ende, artificio. Si José Donoso opone la naturaleza al artificio, Henry James (1973b) opondrá la vida a la novela, puesto que el artista debe producir una "ilusión de vida" (19), "una visión de las cosas" (19) y una "impresión de realidad" (25). De esta manera, la vida, la realidad o las cosas serán convertidas en artificio en la medida que una conciencia observadora las represente: "Puesto que la vida es toda inclusión y confusión, y en el arte todo discriminación y selección, este último, en busca del recio valor oculto que es el único que le concierne, olfatea la masa tan instintiva y certeramente como un perro que barrunta un hueso enterrado" (James, 1975: 63).

El segundo lugar, la casa para Donoso adquiere importancia en términos de composición narrativa en la medida que se concibe como el recurso con el cual se lleva a cabo la plasmación de los personajes o del personaje como sujeto de la mirada. $\mathrm{Al}$ recordar aquellas casas que han sido importantes para su vida, el autor de Casa de campo (1979) afirma en el prólogo invertido de El obsceno pájaro de la noche $e^{5}$

Casas inolvidables, sobre todo, porque de alguna manera encarnaban un lapso de vida específico: cada una era como una novela. No porque tuviera nada de novelesco lo que allí aconteciera, sino porque en cada una de ellas los personajes -yo entre ellos- tomábamos una coloración específica, in-

\footnotetext{
${ }^{5}$ Me refiero al texto de José Donoso "Claves de un delirio" que se incluyó como apéndice en una edición de El obsceno pájaro de la noche del año 1997. Es interesante destacar las similitudes e inversiones con respecto al modus operandi jamesiano que este texto desencadena. En primer lugar, es un texto que explica póstumamente el proceso creativo de su novela maestra diecisiete años después de escribirla y publicarla, así como Henry James, ya casi al final de sus días, escribió los prólogos a una gran cantidad de sus obras cuando preparaba la edición de Nueva York. Por otro lado, Donoso juega con la idea del prólogo jamesiano, ya que, si bien su texto, al igual que los del maestro norteamericano, explica el origen y la composición de sus ficciones, no funciona como prólogo, sino como apéndice. Es, desde luego, un prólogo invertido, porque no antecede la novela, sino que la sigue.
} 
confundible. Entonces, todos los personajes pertenecían a alguno de esos diversos mundos indestructibles: la palabra casa y la palabra novela son una y la misma para mí (Donoso, 1998b: 574).

Llama la atención cómo José Donoso hace uso de recursos pictóricos y visuales para describir y comprender la novela, tal como James echa mano de la arquitectura y de la pintura para justificar por sí misma la novela. En efecto, la analogía entre pintura y novela permite al escritor norteamericano criticar a aquellos que, desdeñando una preocupación por la forma, le exigen al arte narrativo una función apologética: "No pienses demasiado sobre el optimismo y pesimismo; esfuérzate y coge el color de la vida misma" (James, 1973a: 33) (el subrayado es mío).

Cabría preguntarse a qué alude J. Donoso cuando habla de la coloración que adquiere el sujeto al habitar una casa. Aquí, la casa no sería una expansión de un yo esencial y original, sino más bien el escenario sobre el cual el sujeto actúa, adquiriendo entonces su identidad tan precaria como un disfraz. Pero también la casa es el lugar de la luz filtrada, de una luz dirigida y regulada. Una casa llena de ventanas, como la casa de la ficción descrita por Henry James, es un espacio luminoso, un espacio al cual la luz entra y no de cualquier manera. En un ámbito más local de reflexión sobre la pintura, el artista Adolfo Couve nos recuerda algo ya sabido por casi todos: que las cosas carecen de un color propio, esencial, definitivo y constante a lo largo del tiempo y que, por ende, el color que tiñe la superficie de esas cosas es producto de la luz que irrumpe momentáneamente en el lugar donde esas cosas se encuentran ya sea de una manera definitiva o pasajera ${ }^{6}$.

Lo anterior puede explicar dos constantes de la obra donosiana. En primer lugar, la preferencia por los espacios interiores y cerrados. Por lo general, los personajes son identificados por sus casas o espacios íntimos. El paisaje en la obra de José Donoso es escaso y la ciudad, si bien llega a tener una enorme importancia, se presenta como una derivación opuesta del espacio doméstico. En segundo lugar, el sujeto, quien es en Donoso un sujeto del encierro, se construye como personaje en su relación con el mundo exterior, relación siempre mediada por la ventana a través de la cual se mira. Por medio de la ventana u otro tajo, lo otro ha irrumpido en el espacio interior. Luego, el personaje, reaccionando, ha gritado y con ese grito se ha construido el $y o$. De este modo, la ventana presenta un doble tránsito: la mirada que

\footnotetext{
${ }^{6}$ Ver Warken, Cristián. 1997. La belleza del pensar. Entrevista a Adolfo Couve. Santiago: Fíl-
} mico. 
se despliega desde dentro hacia afuera y, por otro lado, la luz externa que irrumpe en el interior, dotando al sujeto observador de cierta coloración tan transitoria como la vida misma.

Por último, aquel sujeto que ha sido coloreado por la luz proveniente del exterior pertenece, en palabras de Donoso, a un mundo indestructible. Considerando el contexto de la cita, creo que ese mundo debe entenderse, en tanto ficción, una realidad que al estar más allá de la nuestra se vuelve invulnerable. Así, la ventana, la luz y el color posibilitan la aparición del sujeto que se diluye más allá del límite de la ficción.

\section{LA MIRADA EXTRAVIADA}

Múltiples son las miradas desde una ventana en el mundo de Donoso. Pienso, por ejemplo, en La misteriosa desaparición de la marquesita de Loria (1980) donde la protagonista se asoma por su ventana para poder identificar, en medio de la noche, el origen de los ladridos callejeros. En El jardín de al lado (1981), las ventanas también adquirirán mucha relevancia, ya que le permiten al protagonista, un pintor chileno autoexiliado llamado Julio Méndez, contemplar el jardín del duque desde el departamento madrileño donde pasa el verano. Llama la atención cómo Ricardo Gutiérrez Mouat ha advertido una vinculación entre esta novela y el prólogo de Henry James a Retrato de una dama. Aquí, el autor norteamericano confiesa que la escritura de la novela le tomó un largo tiempo y recuerda que, estando en Venecia, la vida de la ciudad que entraba por su ventana lo distraía de su trabajo. Julio Méndez, de igual modo, se distrae de su escritura por la bellísima vista al jardín de al lado que la ventana le provee:

En el prólogo a Retrato de una dama James escribe que la forma literaria se construye mediante el encuadramiento de la visión artística como si ésta se percibiera a través de una ventana. Su visión retrospectiva de esa novela echa una extraña luz sobre El jardín de al lado, de Donoso... El autor dramatizado de la novela donosiana se cambia de domicilio para escribir su novela y también se deja distraer por los rumores de las cercanías mientras trata, inútilmente, de completar la versión final del texto. El lago veneciano se convierte en el jardín de al lado donosiano, y las visiones que toman cuerpo en este aristocrático paraje se dejan invadir por el rumor de la memoria y por las visiones de otro jardín añorado (Gutiérrez, 2006: 11-12). 
Pienso también en El lugar sin límites (1966), novela en la que don Alejo, el terrateniente que domina política y económicamente al pueblo El Olivo, mirará por la ventana la simulada relación sexual de la Japonesa y de la Manuela. Finalmente, la cabrona y el travestí del prostíbulo llevan a cabo la relación sexual que termina siendo tan verdadera como la muerte misma y que convierte al patrón en el perdedor de la misma apuesta que inventó.

Sin embargo, de todos los pasajes donosianos en los que la ventana y la casa figuran, me gustaría detenerme a continuación en el de El obsceno pájaro de la noche (1970) donde Iris, la huérfana de la Casa de los Ejercicios Espirituales de la Encarnación de la Chimba, aparece sobre el alféizar de la ventana abarrotada del segundo piso para bailar grotescamente ante la mirada de los muchachos del barrio. Su baile, solicitado y aclamado por ellos, está dirigido a Romualdo, el chiquillo que se le conoce como el "Gigante", porque reparte volantes publicitarios con una máscara de cartón que le cubre totalmente la cabeza. Iris mantiene relaciones sexuales con este personaje, desconociendo la identidad existente tras esa cabeza de cartón. Es decir, para Iris no hay otro más que el Gigante. La muchacha también disfraza con inocencia las relaciones sexuales que sostiene con él, denominándolas infantilmente como "nanay". Son varios los aspectos de este pasaje que quiero destacar porque guardan relación con la ficción y con la representación temática de una mirada obstaculizada y desplegada desde la casa.

En primer lugar, la ventana en la cual Iris se encarama tiene barrotes y gran parte de la superficie de sus vidrios están pintados para impedir la visión: "Dos ventanas idénticas: altas, angostas, alféizar amplio, vidrios pintados color chocolate hasta la altura de una persona para que nadie vaya a ver lo que hay afuera salvo esos nubarrones velados por la rejilla metálica y los barrotes" (Donoso, 1998b: 28). Es decir, la mirada de Iris y de las otras huérfanas que se asoman a las ventanas está repetidas veces interferida por una serie de pantallas o filtros que deforman el espectáculo callejero. Desde el interior, en el cual los personajes femeninos permanecemos encerrados, el vidrio, la pintura, la rejilla metálica y los barrotes simultáneamente censuran y nos permiten ver, junto a las niñas, lo que acontece más allá de la ventana.

El encierro funciona aquí como una isotopía que construye el modo de mirar y lo mirado, porque, al ser fuente de insatisfacción, precariedad y aburrimiento, desplaza una y otra vez el deseo, manteniéndolo vigente. En efecto, Iris mira y baila en el alféizar de la ventana al estar motivada por el deseo, cuyo objeto lo identificamos, en primer lugar, con los "nanay", es decir, con las relaciones sexuales que mantiene con el Gigante por medio 
de quien la niña obtiene una serie de bienes tales como dinero, revistas y maquillajes, es decir, recursos que posibilitan una transformación, la construcción de una nueva identidad y la tachadura del yo pasado que se vuelve un otro. La mirada de Iris está dirigida directamente a los ojos del joven, única parte del rostro de Romualdo que la cabeza de cartón del Gigante permite ver por medio de unos orificios. Por ende, el relato presenta otro filtro más que la mirada debe sortear. Tras el vidrio, la pintura color café, la rejilla metálica, los barrotes y el cartón de la máscara, Iris ve los ojos de quien desea, objeto con cuyo vínculo visual se vuelve lo que en ese momento llega a ser fugazmente: Gina, un personaje de Corín Tellado, esa bailarina aclamada y famosa que vivía en un convento de monjas y no una huérfana con problemas de retraso intelectual:

Se prende de los barrotes para mirarlo. Es él, los ojos redondos del porte de dos platos, la risa que no cambia porque nunca se enoja, él es bueno, hacemos nanay rico y me dice Gina, la ceja arqueada que sujeta con las arrugas de su frente el ridículo sombrerito... es él, se quiere casar conmigo porque le gusta como hago nanay, me va a llevar a ver películas donde las artistas se mueven solas y hablan sin que la pesada de la Eliana me tenga que estar leyendo nada, el gigante me va a llevar a uno de esos edificios altos que se ven allá en el centro para que yo baile en un concurso y me den el premio, pinturas para la cara dicen que le dan a la chiquilla que baila mejor y después la sacan retratada en todas las novelas y la tonta de la Eliana y la señora Rita y el Mudito y la Madre Benita y las chiquillas y todas las viejas me van a ver retratada en las novelas cuando yo salga (Donoso, 1998b: 31).

La mirada permite entonces al personaje figurar como ficción, recurso que ya había sido anticipado con las revistas de historietas del Pato Donald que Romualdo le entrega a Iris a cambio de sexo. La presentación donosiana del sujeto más como un disfraz performático que como una identidad fundada en una esencia es recalcada con los cirios que iluminan la ventana y que posibilitan que Iris se vuelva un objeto de las miradas callejeras y, por ende, un espectáculo del anochecer. Eliana ha robado dos cirios de la capilla de la Casa de Ejercicios Espirituales para convertir la ventana en un escenario:

Queda sola en la ventana iluminada. Quiebra la cadera. Adelantando los pechos se ciñe el suéter con una larga caricia que recorre todo su cuerpo y termina arremangándose la pollera para mostrar los muslos gruesos, de masa vibrante, mientras con la otra mano se sube el pelo, frunciendo los 
labios como para besar con loca pasión. En la calle, el grupo que se va juntando debajo del farol aplaude (Donoso, 1998c: 30).

La ventana como escenario también se invierte, derivando en un altar en el que la representación de Iris-Gina simula ser una Virgen a la luz de las velas devotas, identificación que volverá a ser tratada en la novela puesto que las viejas de la Casa inventarán que el supuesto embarazo de la Iris, al no conocer varón, es un milagro:

Gina, mijita, échale no más mijita linda, que se te muevan harto las tetas, que se desarme el poto, que arda la Casa, que ardamos todos. Y el Gigante, con su enorme cabezota de cartonpiedra, sale al medio de la calle a bailar como si bailara con la Iris, la Iris se cimbra, mueve su cintura y gira y se agita y chilla allá arriba encerrada en su jaula iluminada por los cirios, suspendida en el flanco de la Casa, bailando como una Virgen que se hubiera vuelto loca en su hornacina (Donoso, 1998b: 30).

Hay un último aspecto de este pasaje que vale la pena resaltar. El vínculo entre el espacio interior y el exterior, la calle, se complejiza al incorporar un tercer elemento que juega como espejo de la ventana en la que Iris se asoma. Se trata de un balcón que encontramos justo al frente de la ventana abarrotada, al otro lado de la calle:

En la casa de enfrente una mujer abre su balcón. Mientras se peina el pelo largo y retinto, mirando la calle, pone la radio, rat-tat-tat-tatatat-tat-tatat, estridencias sincopadas de guitarras eléctricas y voces gangosas invaden el dormitorio, levantan a la Iris del somier, la ponen de pie en el pasillo entre las dos hileras de catres al oír babalú, babalú ayé, ya échanos un bailecito, Gina, la animan las huerfanitas, échale no más, con un gesto de yegua hace caracolear las largas ondas de su pelo... (Donoso, 1998b: 29).

Más allá de la relación especular entre ambas ventanas y sus respectivas mujeres de pelo largo, el balcón amplía el horizonte sensitivo de la mirada hacia la audición. En realidad, Iris, al comienzo, no ha mirado todavía desde el balcón, sino sólo ha oído la música proveniente de él y que la estimula a bailar. Así, el balcón, tal como Henry James lo describe, es un elemento de distracción y estímulo?.

${ }^{7}$ Los balcones también se presentan con frecuencia en la obra de Henry James. Esta extensión 
¿Quién es entonces el que ha visto el balcón? La pregunta alude a la identidad del narrador que, en este pasaje, corresponde al Mudito. Habiendo ya marcado su voz con el pronombre de la primera persona singular al inicio del capítulo, el Mudito no sólo administra el conocimiento narrativo, sino también tiene el poder de focalizarse en Iris hasta el punto de poder reproducir su mirada. Nosotros miramos a través de la mirada del Mudito quien, a su vez, mira como Iris mira. La sucesión de pantallas vuelve la escena sumamente compleja porque Iris no sólo es sujeto de mirada, sino también objeto de observación por parte del Mudito y de nosotros. Desde los ojos de Romualdo hasta los nuestros, la imagen transita, pasa y se cuela por una serie de bastidores y tamices, desencadenando el extravío tanto del objeto original como del sujeto observador en la compleja representación donosiana. La mirada, por ende, se nos ha perdido y con ello nos extraviamos en el lugar desde donde miramos. La casa se nos vuelve entonces algo extraño.

Pienso que los dos puntos que he trabajado, vale decir, el carácter metaliterario de la casa y la representación de tal espacio, tienden a formar una sola unidad. En efecto, la casa como metáfora de ficción coincide con aquello a lo que José Donoso indirectamente está aludiendo en el pasaje de $E l$ obsceno pájaro de la noche que hemos analizado. Aquí, la casa, la ventana y la mirada devienen en la ficción con la cual el sujeto se identifica: Iris es Gina, un personaje inventado por novelas de alto consumo. Por ende, el pasaje aludido también gozaría de un valor metaliterario: una ficción que habla de la ficción o de cuan ficción es o de cuan encerrada permanece dentro de los barrotes de la ficción, porque se trata de una mirada que, al estar interferida, todo lo que toca y mira lo vuelve invención.

Por último, creo que José Donoso sintetiza una posible oposición que distanciaría las posturas de Henry James y Zola en lo que respecta al problema de la mirada interferida u obstaculizada. Si James propone un tránsito desde la conciencia al objeto que se mira, Zola, en cambio, complejiza ese tránsito original al proponer que el objeto que está en el exterior se proyecta en el velo de la ventana, volviéndose la imagen que el sujeto, desde el interior de la casa, mira u observa. Se trataría algo así como la imagen cinematográfica o la diapositiva que proyectamos sobre un telón blanco colgado en la pared. Por su parte, José Donoso, al producir el extravío de la mirada,

de la ventana ya había figurado en Henry James como espacio intermedio entre el interior de la casa y el espacio abierto de la ciudad. "Hay páginas del libro que, al releerlas, me han hecho revivir la severa curva del ancho Riva, las extensas manchas coloreadas de las casas con balcones y las ondulaciones repetidas de los pequeños puentes..." (James, "Prólogo a The portrait of a Lady", p. 54). 
dispone de tres pares de ojos, intensificando el tránsito ya no sólo de una mirada, sino de varias. Los ojos de Romualdo, los ojos de la Iris y los ojos del Mudito hacen estallar el objeto mirado que ya no identificamos, porque el ojo que mira es también el ojo mirado por otro. La relación hasta entonces jerárquica entre un sujeto observador y un objeto observado se ha dislocado $y$, con ello, las identidades con las cuales definíamos ambos elementos se han perdido.

\section{REFERENCIAS}

Bachelard, Gaston. 1975. La poética del espacio. Tradución de Ernestina de Champourcin. México: Fondo de Cultura Económica.

Cozarinsky, Edgardo. 1964. El laberinto de la apariencia. Buenos Aires: Losada.

Donoso, José. 1998a. "El discreto encanto de los prólogos". En Artículos de incierta necesidad. Selección de Cecilia García Huidobro. Santiago: Alfaguara, pp. 394-401.

1998b. El obsceno pájaro de la noche. Santiago: Alfaguara.

Dopico, Ana María. 2001. "Imbunches and Others Monsters: Enemy Legends and Underground Histories in José Donoso and Catalina Parra", en Journal of Latin American Cultural Studies vol. 10, No 3, pp. 325351.

Fazio, Silvina Celeste. 2010. "Figuraciones delegadas: de la inscripción al desplazamiento del yo en Efectos personales de Juan Villoro", en Revista Taller de Letras 46, pp. 21-30.

Gutiérrez Mouat, Ricardo. 2006. "Ensayo preliminar”. En Mascarada. Tres novelas cosmopolitas. En José Donoso. México: Fondo de Cultura Económica, pp. 9-26.

James, Henry. 1973a. "Prefacio a Retrato de una dama". En El arte de la novela y otros ensayos. Traducción de Igor Retamales y Viviana Lutty. Valparaíso: Ediciones Universitarias de Valparaíso, pp. 53-72.

1973b. "El arte de la novela". En El arte de la novela y otros ensayos. Trad. Javier Aguirre. Valparaíso: Ediciones Universitarias de Valparaíso, pp. 7-34.

. 1975. "Prólogo a The Spoils of Poynton". En El futuro de la novela. Traducción y edición de Roberto Yahni. España: Taurus, pp. 62-3.

Ortega, Julio. 2010. "José Donoso: Notas en torno a Lagartija sin cola". En La imaginación crítica. Prácticas de innovación en la narrativa contempo- 
ránea. Santiago de Chile: Ediciones Universidad Alberto Hurtado, pp. 475-77.

Stoichita, Víctor I. 2005. Ver y no ver. La lematización de la mirada en la pintura impresionista. Traducción de Anna María Coderch. España: Siruela.

Valdés, Adriana. 1975. "El "Imbunche". Estudio de un motivo en El obsceno pájaro de la noche". En José Donoso. La destrucción de un mundo. Buenos Aires: Fernando García Cambeiro, pp. 125-160. 